\title{
The deubiquitylating enzyme Cops6 regulates different developmental processes during early zebrafish embryogenesis
}

\author{
WILLIAM K.F. TSE\#,1, MAY-SU YOU2, STEVEN HAO-KEE HO ${ }^{1}$ and YUN-JIN JIANG*,1,2 \\ ${ }^{1}$ Laboratory of Developmental Signalling and Patterning, Genes and Development Division, Institute of \\ Molecular and Cell Biology, Singapore and ${ }^{2}$ Division of Molecular and Genomic Medicine, National Health \\ Research Institutes, Miaoli County, Taiwan
}

\begin{abstract}
Zebrafish cops 6 encodes a putative deubiquitylating enzyme (DUB) that belongs to the JAMM family. It consists of 297 amino acids and includes the Mov34/MPN/PAD-1 (PF01398) domain. Ubiquitylation is involved in many cellular processes and deconjugation of ubiquitinmodified substrates is important to maintain a sufficient amount of free ubiquitin in the cell. Here, we report our findings regarding the general function of the cops 6 gene, as a continuation of our previous studies involving DUB knockdown screening. We have found that cops6 plays different roles in early embryonic development in the zebrafish, including dorsoventral patterning, convergent extension movement and brain formation. In addition, our findings indicate that cops 6 plays an anti-apoptotic role during segmentation. Overall, the present study that consolidates our previous work on zebrafish DUB genes, corroborates the hypothesis of multi-functional roles for DUB genes during development.
\end{abstract}

KEY WORDS: Cops6, deubiquitylating enzyme, zebrafish, vertebrate development, anti-apoptotic factor

\section{Introduction}

Protein modification by ubiquitin (UBQ) and/or ubiquitin-like (UBL) molecules is an important mechanism in regulating numerous critical cellular processes, such as signal transduction, transcriptional control, protein degradation, epigenetic modification and intracellular trafficking. Deconjugation of UBQ and/or UBL substrates is essential to maintain a sufficient free UBQ/UBL pool within the cell. Deubiquitylating enzymes (DUBs) play a key role in these processes (Hershko and Ciechanover, 1998). Recently, our lab performed an in silicogenome-wide search and identified more than 90 putative DUB genes in the zebrafish genome (Tse et al., 2009). Here, we report the results of further research on the the general functions of cops6 (COP9 constitutive photomorphogenic homolog subunit 6) DUB gene, which belongs to the family of JAMM motif proteases (JAMM) family. The JAMM family is the only DUB class that consists of metalloproteases, while all other DUB families (USP, UCHL, OTU and MJD) are cysteine proteases (Nijman et al., 2005). Based on our previous screen, there are 14 JAMM members in the zebrafish genome (Tse et al., 2009). Only a few DUB genes related to disease, such as CYLD (Kovalenko et al., 2003) and ATXN3 (Scheel et al., 2003), have been well studied; the functions of the majority of them remain to be elucidated.

In our previous study, we carried out 85 DUB gene knockdown experiments by means of morpholino (MO) injection and classified the morphants into five groups (GI-GV) according to their huC expression patterns. cops 6 belongs to Group III, indicating that morphants exhibit a decreased and disrupted huC expression pattern (Tse et al., 2009). Based on the earlier findings, we speculated that the cops 6 gene may play important roles in the early development of the zebrafish. Here, we demonstrate the developmental importance of the cops 6 gene. Having characterized the gene and described its expression pattern in zebrafish, we performed functional knockdown studies. On the basis of these results, we propose that cops 6 is required for dorsoventral patterning, convergent extension movement and brain formation and may act as an anti-apoptotic factor during zebrafish development. To our knowledge, this is the first study that characterizes various developmental functions of $\operatorname{cops} 6$, thereby providing evidence of the multi-functional roles of zebrafish DUBs.

Abbreviations used in this paper: Cops6, COP9 constitutive photomorphogenic homolog subunit 6; DUB, deubiquitylating enzyme; UBQ, ubiquitin; UBL, ubiquitin-like.

\footnotetext{
*Address correspondence to: Yun-Jin Jiang, Division of Molecular and Genomic Medicine, National Health Research Institutes, 35 Keyan Road, Zhunan Town, Miaoli County 35053, Taiwan. Fax:+886-37-58-6459. e-mail: yjjiang@nhri.org.tw

"Current address: Laboratory of Physiology, Atmosphere and Ocean Research Institute, The University of Tokyo, 5-1-5 Kashiwa no ha, Kashiwa city, Chiba 277-8564, Japan.
} 
A

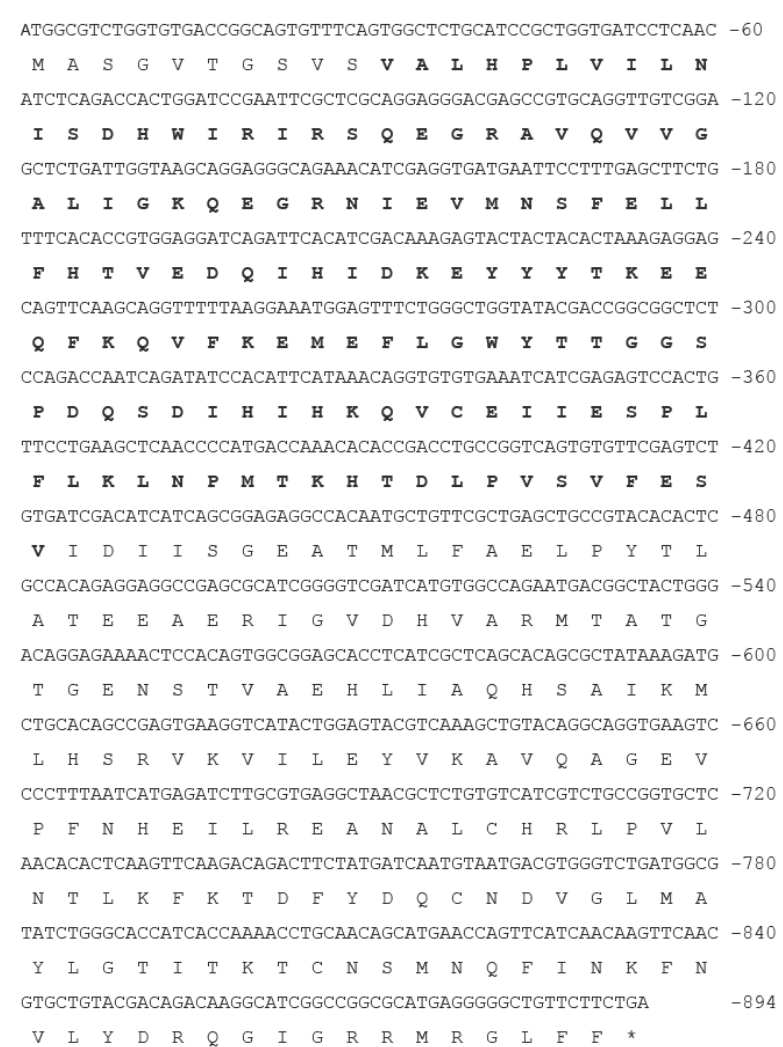

Fig. 1. Cops6 DNA and protein alignments among species. (A) DNA sequence of the 894 bp zebrafish cops6 cDNA and the deduced 297 amino acid sequence. Cops6 belongs to the DUB JAMM family, whose conserved Mov34/MPN/PAD-1 domain is indicated in bold. (B) Comparison of amino acid sequence of the Danio rerio Cops6 (NP_001017768) with those of Xenopus tropicalis (NP_001006723), Mus musculus (AAH14286) and Homo sapiens (NP_006824). The Cops6 sequence is highly conserved (over 85\%) among different species. There is an elongation of amino acids in the mouse and human COPS6s. Asterisks represent 100\% identity; colons indicate $75 \%$ identity, including zebrafish sequence; periods indicate 50-75\% identity, excluding the zebrafish sequence. (C) Protein alignments between Cops6 and 13 other DUB JAMM members in zebrafish. Eif3f (XP 685399) and Psmd7 (NP 956083) showed highest similarity to Cops6. However, they only have about 20\% identity with Cops6, which mainly locate in the JAMM domain region. This result suggests that JAMM DUBs may not have evolved in parallel. The analysis was performed by Clustal V method using the Megalign program of Lasergene 6.

\section{Results}

\section{The Cops 6 protein contains the conserved JAMM domain} cops 6 is a DUB gene that belongs to the JAMM family. It has 297 amino acids, including the Mov34/MPN/PAD-1 (PF01398) domain (Fig. 1A). The domain is evolutionarily conserved among different species. Zebrafish Cops 6 exhibits high sequence similarity (over $85 \%$ identity) among different species, from frog to human. An elongation of amino acids near the N-terminal occurred during evolution; thus, there are 27 amino acids more in mice and 30 more in humans. Nevertheless, the JAMM domain remains highly conserved (Fig. 1B). Protein alignments among zebrafish Cops6 and other JAMM DUB members revealed that two JAMM members, Psmd7 and Eif3f, share the highest similarity with Cops6 (Fig. 1C).
B Xeno tome

Danio Xeno Mus FLGWYTTGGTPDPSDTHVHKOUCETTESPIFIKTNPMTKHTDI PVSVYESVTDTVNGEAT 150 FLGWYTTGGPPDPSDIHVHKOVCETIESPLFLKLNPMTKHTDL PVSVFESVIDTINGEAT 177 FLGWYTTGGPPDPSDIHVHKQVCEIIESPLFLKLNPMTKHTDLPVSVFESVIDIINGEAT 180

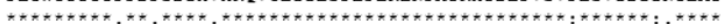

DaniO MLFAELPYTLATEEAERIGVDHVARMTATGTGENSTVAEHLIAQHSAIKMLHSRVKVILE 210 Xeno MLLAELSYTLATEEAERIGVDHVARMTATGSGENSTVAEHLIAQHSAIKMLHSRVRLILE 210 MUS MLFAELTYTLATEEAERIGVDHVARMTATGSGENSTVAEHLIAOHSAIKMLHSRVKLILE 237 Homo MLFAELTYTLATEEAERIGVDHVARMTATGSGENSTVAEHLIAQHSAIKMLHSRVKLILE 240

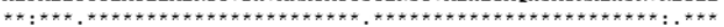

DaniO YVKAVQAGEVPFNHEILREANALCHRLPVLNTLKFKTDFYDOCNDVGLMAYLGTITKTCN 270 Xeno YVRAAEAGEVPFNHEILREASALCHCLPVLSTDKFKMDFYDQCNDVGLMSYLGTITKTCN 270 Mus YVKASEAGEVPFNHEILREAYALCHCLPVLSTDKFKTDFYDQCNDVGLMAYLGTITKTCN 297 YVKASEAGEVPFNHETLREAYALCHCLPVLSTDKFKTDFYDOCNDVGLMAYLGTITKTCN 300 YVKASEAGEVPFNHEILREAYALCHCLPVLSTDKFKTDFYDQCNDVGLMAYLGTITKTCN 300

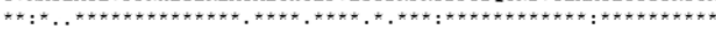

Danio SMNQFINKFNVLYDRQGIGRRMRGLFF 297 Xeno TMNQFVNKFNILYDRQGIGRRMRGLFF 297 Mus TMNQFVNKFNVLYDRQGIGRRMRGLFF 324 TMNOFVNKFNVLYDROGIGRRMRGLFF 327

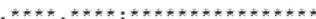
MACGVIGSVSVALHPLVILNISDHWIRMRS 60

QEGRUQUTGAT GGRPVQVIGALIGKQEGRNIEVMNSFELLSQINEEKITINKEYYYTKEEQFKQVFKDME 90 QEGRPMQVIGALIGKQEGRNIEVMNSFELLSHTVEEKIIIDKEYYYTKEEQFKQVFKELE 117 QEGRPVQVIGALIGKQEGRNIEVMNSFELLSHTVEEKIIIDKEYYYTKEEQFKQVFKELE 120 -MAAAAAGANGSGGSSGMEVDAA-VPSVMASGVTGSVSVALHPLVILNISDHWIRMRS 57 
A
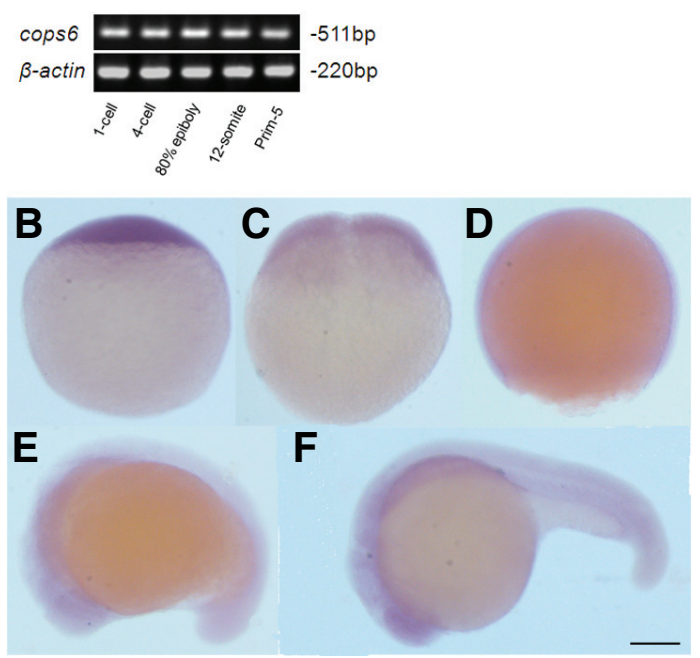

Fig. 2. Zebrafish cops 6 expression at early developmental stages. cops6 started its expression at the 1-cell stage and was ubiquitously expressed throughout early developmental stages. (A) RT-PCR results. (B-F) In situ hybridization results of embryos at different developmental stages: (B) 1-cell; (C) 4-cell; (D) 80\% epiboly; (E) 12-somite and (F) prim5. Throughout early development, cops 6 mRNA was expressed at similar levels and no specific expression pattern was observed. Scale bar: 150 $\mu m(\mathrm{~B}-\mathrm{F})$.

cops 6 mRNA into single-cell embryos. However, no significant phenotypic changes were observed (Table 1). Next we used MO knockdown to study its loss-of-function effect. Over $80 \%$ of cops 6 morphants exhibited C3-C4 dorsalized phenotypes (Table 1), which consisted of the loss of the posterior regions during development (Fig. 3 A-F). In addition, rescue experiments were performed to address MO specificity. The knockdown effect of MO1, which targets ATG region, was rescued by cops $6 \mathrm{mRNA}$ co-injection (Table 1; Fig. 3 A-I).

Phenotypes were further examined and verified by using a panel of in situmolecular markers at different stages. At 60-75\% epiboly stage, one dorsal marker (chd) and one ventral marker (eve1) were used. Expression of $c h d$ is dorsally restricted, while eve 1 is expressed in the ventrolateral marginal cells. cops6 morphants exhibited an expanded chd expression pattern and a contracted eve1 expression pattern (Fig. 4 A-D). In addition, we examined $n t /$ and $d / x 3$ expression by in situ hybridization to

TABLE 1

\section{PHENOTYPIC FREQUENCY OF RNA AND/OR MO INJECTION EXPERIMENTS}

\begin{tabular}{ccccccccccc} 
RNA/MO & $\begin{array}{c}\text { pg/em } \\
\text { (RNA) }\end{array}$ & $\begin{array}{c}\text { pmol } \\
\text { (MO) }\end{array}$ & n ep & n & C5 \% & C4 \% & C3 \% & C2 \% & C1 \% & WT \% \\
\hline cops6-MO1 & & 1.5 & 4 & 152 & 43 & 42 & 15 & & \\
cops6-MO2 & & 2.5 & 2 & 88 & 33 & 32 & 23 & 12 & \\
cops6-mRNA & 600 & & 2 & 53 & & & & & 100 \\
cops6-mRNA+ & 600 & 1.5 & 3 & 103 & & & & 17 & 83 \\
cops6-MO1 & & & & & & & & & &
\end{tabular}

Two cops6MOs were used to test the knockdown specificity. MO1, which targets the ATG site showed a better knockdown efficiency than MO2 that targets the 5'-UTR. In addition, co-injection of cops 6 mRNA and cops 6 -MO1 could rescue over $80 \%$ of the dorsalized phenotype of morphants, indicating that cops6-MO1 knockdown is specific. Phenotypic frequency is indicated in the table. C1-C5 phenotypes represent dorsalized phenotypes as described in (Mullins et al., 1996). Abbreviations: em, embryo; $n$ ep, number of experiments; $n$, number of scored embryos. identify if knockdown causes aberrant convergent extension (CE) defects (Topczewski et al., 2001). We found that the axial expression of $n t /$ became broader at $60-75 \%$ epiboly (data not shown), while the $d / x 3$ expression domain (neural plate boundary) expanded at the 1-4 somite stage (Fig. $4 \mathrm{E}-\mathrm{F}$ ), indicating that $\mathrm{CE}$ movement is also affected.

When the embryo reached the $12-14$ somite stage, widening of the somite muscle in morphants became obvious (Fig. $4 \mathrm{G}-\mathrm{H}$ ). This finding was corroborated using the somite marker, myoD. Thus, in morphants, reduced, diffused and laterally-expanded myoD expression was detected (Fig. 4 I-J). Using pax2a, which labels the presumptive neural region (Krauss et al., 1991), we found that morphants exhibited widening of the trunk with increased lateral distance between otic vesicles and pronephroi (Fig. $4 \mathrm{~K}-\mathrm{L}$ ). Furthermore, shortening of the anteroposterior embryonic axis and a reduction of the longitudinal distance between the mid-hindbrain boundary and otic vesicles were observed in morphants (Fig. 4 K-L).

\section{cops 6 is critical in early zebrafish brain development}

cops6 has been classified into Group III on the basis of in situ huC screening, indicating that its morphants have fewer and unorganized neurons (Tse et al., 2009). In this study, we further investigated its role in brain development. Brain regionalization, which is an important step for brain development, involves the action of a variety of transcription factors such as Krox20, Otx2, Pax2and Eng2(Joyner and Guillemot, 1994). In addition, the midhindbrain region has been suggested to be an organizing center for midbrain patterning and induction (Marin and Puelles, 1994) and its boundary (MHB) is important in restricting cell lineage

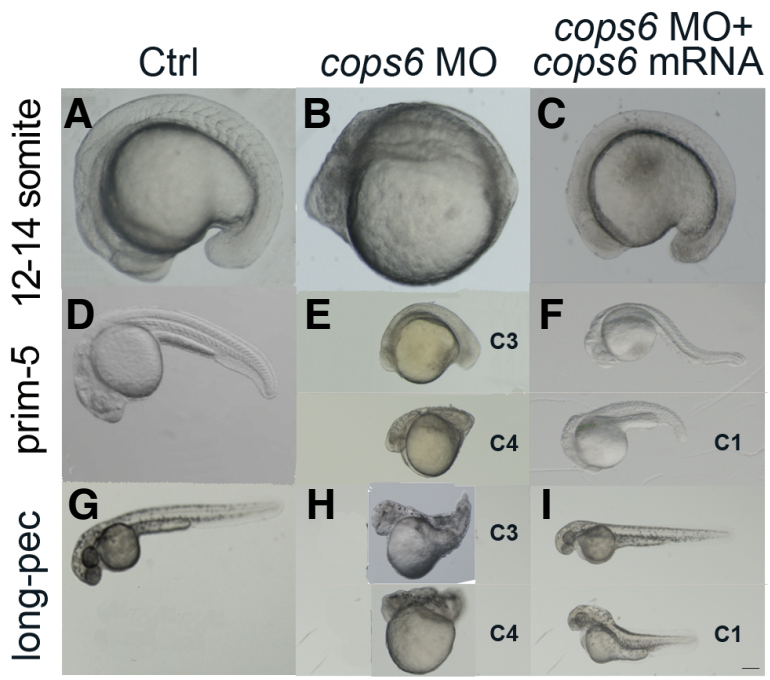

Fig. 3. Phenotypes of cops 6 morphants. cops 6 morphants in an $A B$ wild-type background showed dorsalized phenotypes at different stages: (B) 12-14 somite, (E) prim-5 and (H) long-pec. They all showed the reduced development of the posterior part, which is a feature of dorsalization. $\mathrm{C} 3$ and $\mathrm{C} 4$ dorsalized phenotypes are shown in $\mathbf{E}, \mathbf{H})$. The dorsalized phenotype caused by cops 6 knockdown was rescued by coinjection of cops 6 mRNA with MO (C,F,I). More than $80 \%$ of dorsalized embryos were rescued back to a phenotype similar to wild-type, while the remaining ones showed a much milder phenotype, C1, in (F,I). Scale bar: $100 \mu \mathrm{m}(A, B), 120 \mu \mathrm{m}(C), 80 \mu \mathrm{m}(D), 150 \mu \mathrm{m}(E, F, I), 90 \mu \mathrm{m}(G)$ and 135 $\mu m(H)$ 

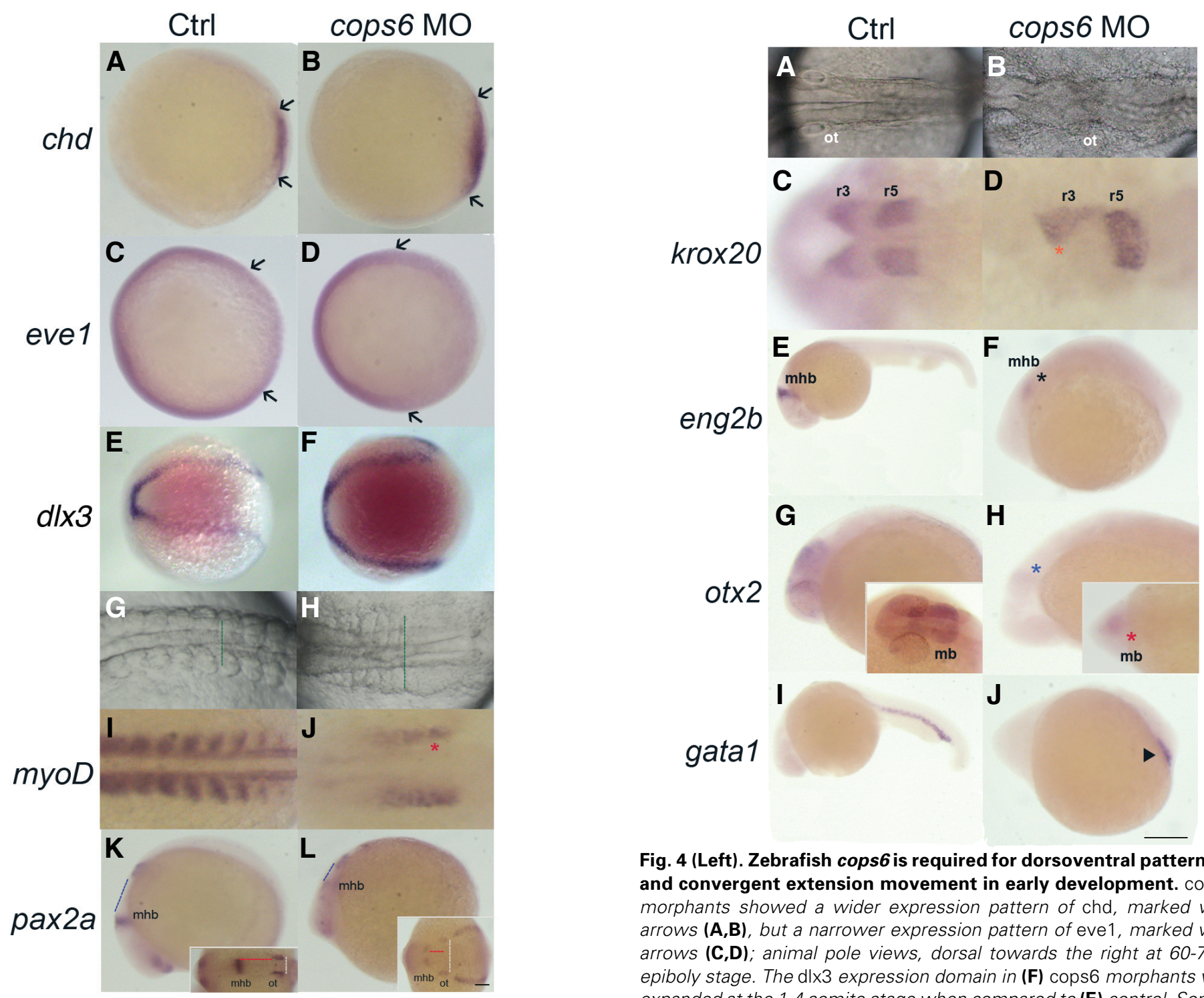

Fig. 4 (Left). Zebrafish cops6 is required for dorsoventral patterning and convergent extension movement in early development. cops 6 morphants showed a wider expression pattern of chd, marked with arrows (A,B), but a narrower expression pattern of eve1, marked with arrows (C,D); animal pole views, dorsal towards the right at $60-75 \%$ epiboly stage. The dlx3 expression domain in (F) cops6 morphants was expanded at the 1-4 somite stage when compared to (E) control. Somite morphology of (G) wild-type and (H) cops6 morphants at the 12-14 somite stage. Lateral expansion of somite muscles was apparent (green dot lines). Furthermore, myoD expression in (J) cops6 morphants was widened and severely disrupted (red asterisk) when compared to (I) control. pax2a expression at the 12-14 somite stage $(\mathbf{K}, \mathbf{L})$ lateral view and $(K-L$, insert) dorsal view. Blue dotted lines represent the distance between the midhindbrain boundary and otic vesicles in the lateral view; while red dotted lines represent this distance in the dorsal view. Shortening of the lateral distance was found in cops6 morphants. On the other hand, white dotted lines indicated the distance between the two otic vesicles, where lengthening of the ventral distance was found in cops6 morphants. mhb: mid-hindbrain boundary; ot: otic vesicle. In all photos, the head is to the left. Scale bar: $70 \mu \mathrm{m}$ (A-D and G-J), $25 \mu \mathrm{m}(\mathrm{E}, \mathrm{F})$ and $150 \mu \mathrm{m}(\mathrm{K}, \mathrm{L})$.

Fig. 5 (Right). Zebrafish cops6 plays a role in brain development, but not in blood vessel formation. (A,B) cops 6 morphants in an $A B$ wild-type background showed a brain defect at the prim-5 stage. The hindbrain was not well formed in (B) cops6 morphants when compared to (A) control; dorsal view. (C,D) Expression of krox20, a marker of rhombomeres three and five; dorsal view. Reduced size in rhombomere three (orange asterisk) and fused rhombomere five expression due to unfolded hindbrain were found. (E,F) eng2b expression that indicates the mid-hindbrain boundary (mhb) was reduced in cops6 morphants (black asterisk). (F) cops6 morphants had a smaller mhb when compared to (E) control; lateral view. (G,H) otx2 expression was reduced in cops6 morphants (blue asterisk in lateral view; red asterisk in dorsal view). The size of the midbrain was decreased in (H) cops6 morphants in comparison to (G) control. (I,J) Presumptive blood marker (gata1) did not show any significant differences between (I) control and $(\mathbf{J})$ cops6 morphants. They both form a normal blood island (triangle mark), which suggests that cops 6 is not required for blood development. mb: midbrain; mhb: mid-hindbrain boundary; ot: otic vesicle; $13 / r 5$ : rhombomere three/five. All photos are with head to the left. Scale bar: 48 um (A, B), $100 \mu m(C, D), 265 \mu m(E, I), 150 \mu m(F, J)$ and $170 \mu m(G, H)$.

(Keynes and Krumlauf, 1994). We collected embryos at the prim5 stage in which the essential brain morphology has been formed in zebrafish.
The hindbrain develops a series of rhombomeres along the anteroposterior axis of the neural tube. This morphological segmentation is visible in the zebrafish at the 18-somite stage (Moens 
and Prince, 2002). Knockdown of the cosp 6 gene resulted in abnormal brain morphology (Fig. 5 A-B) and aberrant krox20 expression (unfolded $\mathrm{r} 5$ and reduced $\mathrm{r} 3$ ) at the prim- 5 stage (Fig. $5 \mathrm{C}-\mathrm{D}$ ), indicating a disturbance in the brain development processes. In addition, eng $2 b$ was used to examine the MHB structure. cops 6 morphants showed a reduced $\mathrm{MHB}$ size. eng2b was expressed in a much more restricted area in morphants in comparison to controls (Fig. $5 \mathrm{E}-\mathrm{F}$ ). Abnormal MHB patterning at an early developmental stage (12-14 somite stage) could also be observed by pax2a expression, which revealed an obvious gap in the MHB in morphants (Fig. $4 \mathrm{~K}-\mathrm{L}$ ). Finally, in order to verify if the midbrain structure is affected in cops 6 morphants, we examined otx2 expression. otx 2 expression was greatly reduced in morphants, indicating a loss or reduction of the midbrain region (Fig. $5 \mathrm{G}-\mathrm{H}$ ). In order to verify if the development of other mesodermal components is altered, we examined gata 1 expression and found no obvious expression change in the presumptive blood region in morphants, suggesting that blood development is not affected in cops6 morphants (Fig. $5 \mathrm{l}-\mathrm{J}$ ).

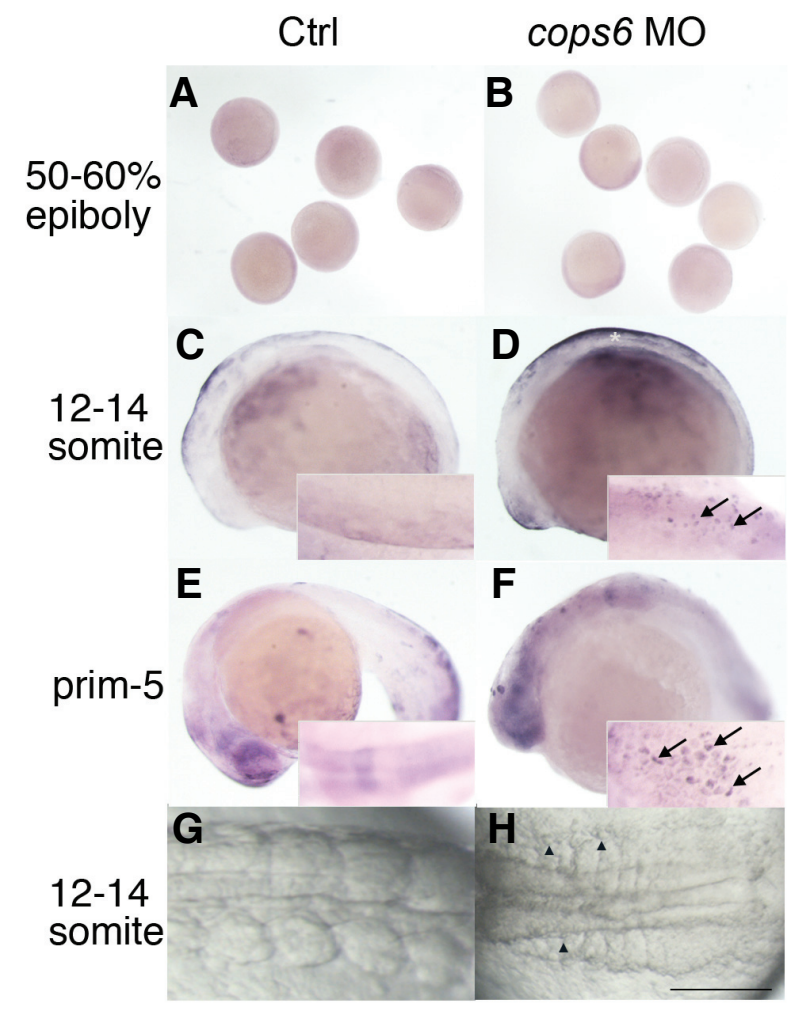

Fig. 6. Zebrafish cops6 plays an anti-apoptotic role in early development. TUNEL assay of wild-type embryos and cops6 morphants at different developmental stages. There were no significant differences between (A) control and (B) cops6 morphants at 50-60\% epiboly stage. At the 12-14 somite stage, (D) cosp6 morphants showed increased positive staining of apoptotic cells in the trunk region (white asterisk) when compared to (C) control; lateral view. Apoptotic cells (arrows) at the same stage, dorsal view (insert), were found in (D) cops6 morphants, but not in (C) control. Afterwards, at (E,F) prim-5 stage, increased numbers of apoptosis-positive stained cells (arrows) were found only in (F) cops6 morphants. (G,H) At the 12-14 somite stage, the apoptotic dying cells, exhibiting a rounded-up cell body (arrowheads), were found in (H) cops6 morphants. Scale bar: $1250 \mu \mathrm{m}(\mathrm{A}, \mathrm{B}), 210 \mu \mathrm{m}(\mathrm{C}-\mathrm{F})$ and $150 \mu \mathrm{m}(\mathrm{G}, \mathrm{H})$.

\section{Cops6 plays an anti-apoptotic role during segmentation}

On the basis of morphological observations, we suspected that cops 6 morphants may undergo increased apoptosis. During the early stage of development, morphology of cops6morphants did not show any significant differences and no obvious apoptotic cells were observed (Fig. 6 A-B). Starting from 12-14 somite stage, we observed an obvious increase in the number of TUNELpositive apoptotic cells in the trunk region and the number of dying cells was apparently increased at the prim-5 stage (Fig. 6 C-F). Furthermore, rounded-up cells, a feature of dying cells, were found in the trunk region of morphants (Fig. $6 \mathrm{G}-\mathrm{H}$ ), supporting the idea that Cops6 may have anti-apoptotic functions during early development.

\section{Discussion}

Zebrafish deubiquitylating enzyme, Cops6, is highly conserved in evolution. It consists of 297 amino acids with the Mov34/MPN/PAD1 domain. In this study, we characterize the zebrafish cops 6 gene and provide evidence of its multi-functional roles in early zebrafish embryonic development. Knockdown of cops 6 results in defects in dorsoventral patterning, CE movement and brain development. In addition, our results point to an anti-apoptotic role for this enzyme in the developing embryos. Hence, Cops6 exerts multiple functions during zebrafish development.

Overexpression of cops 6 was not found to cause obvious morphological changes. Cases have been reported that gene overexpression does not lead to any phenotypic changes (Huang et al., 2007). Nevertheless, the co-injection of cops $6 \mathrm{mRNA}$ with its MO rescued $\mathrm{MO}$-induced phenotypes, indicating that cops $6 \mathrm{mRNA}$ is translated and functional (Table 1). This result may supports our previous finding that DUB genes can have redundant roles. Therefore, single knockdown of group IV DUB cannot compensate the effect of bmp4 overexpression (Tse et al., 2009).

It should be noted that the deubiquitylating activity of zebrafish Cops6 has not been biochemically demonstrated. Nevertheless, Cops6 is generally classified as a DUB member, thanks to its Mov34/ MPN/PAD-1 domain, which has been shown to cleave the UBL protein, Nedd8, from the Cul1 subunit of SCF ubiquitin E3 ligases (Cope et al., 2002). Thus, the DUB activity of Cops6 needs to be confirmed, and the substrates of this enzyme also need to be identified. In the light of the fact that its depletion results in multiple defects during zebrafish development, it is conceivable that Cops6 has several substrates that are required for corresponding developmental processes. Furthermore, a genomic search has revealed that the number of ubiquitin E3 ligases is far higher than that of DUBs (Nijman et al., 2005; Tse et al., 2009), which indicates that in general DUBs may have more substrates than E3 ligases. To better understand the function of Cops6 in zebrafish development, it will be necessary to identify its substrates in the future.

\section{Materials and Methods}

\section{Fish strains and maintenance}

The strain used in this study was the $A B$ wild-type line. They were raised and staged as previously described (Kimmel et al., 1995). All experimental procedures on zebrafish embryos were approved by the Biological Research Centre, A*STAR, Singapore (BRC IACUC No. 080390) and the Institutional Animal Care and Use Committee, National Health Research Institutes, Taiwan (NHRI-IACUC-098018). 


\section{Morpholino (MO) sequence site selection, design and specificity}

The translation initiation site (TIS) of the Cops 6 protein sequence was located by BLAST (http://blast.ncbi.nlm.nih.gov/Blast.cgi, Altschul et al., 1997), and all potential upstream sequences for MO target oligos were processed with AMOD (Klee et al., 2005). Antisense MOs were selected based on the guidelines from Gene Tools (reviewed by Eisen and Smith, 2008). In addition to MO-1, targeting the ATG site (5'CGGTCACACCAGACGCCATCACACT-3'), MO-2, targeting 5'-UTR (5'GGCTCGCTGAACAGAAGAGTGGAGA-3'), was generated to confirm the knockdown phenotypes. Furthermore, cops 6 mRNA rescue experiments were performed to verify the knockdown specificity (Table 1). In addition, the MO-induced phenotypes were not associated with those non-specific effects caused by overdose MO treatment (unpublished observations). Unless specified, all the results were generated by MO-1.

\section{Expression construct generation and mRNA in situ probe synthesis cops6 was amplified with primers:}

5'-CCGAATTCATGGCGTCTGGTGTGA-3' and

5'-CCCTCGAGTCAGAAGAACAGCCC-3', containing EcoRI and Xhol restriction sites respectively, from full-length cDNA using PfuDNA polymerase (Stratagene) and ligated into $\mathrm{pCS} 2+$ to generate the $\mathrm{pCS} 2+\operatorname{cops} 6$ expression construct. The construct vector was linearized by Notl; capped RNA was synthesized with the SP6 Message Machine kit (Ambion) and finally dissolved in DEPC-treated water. Antisense RNA probe was synthesized by using digoxigenin RNA labeling mix (Roche) and T7 polymerase (Promega).

\section{Morpholino (MO) and mRNA injection}

All MOs were purchased from Gene Tools, re-suspended in distilled water to make a $5 \mathrm{mM}$ stock and stored at $-20^{\circ} \mathrm{C}$. Diluted MOs and/or mRNA (amount listed in Table 1) were injected into one- or two-cell stage embryos. Embryos from four different pairs of fish were used for each MO and/or mRNA injection.

\section{Whole-mount in situ hybridization (WISH)}

Plasmids that were used to make antisense mRNA probes for in situ hybridization have been previously described elsewhere (Tse etal., 2009; Ma and Jiang, 2007).

\section{Detection of apoptotic cell death (TUNEL Assay)}

Apoptotic cell death in zebrafish was detected according to the manufacturer's protocol (In situ Cell Death Detection Kit-AP; Roche).

\section{RNA extraction, reverse transcription and RT-PCR}

Embryos at different developmental stages were collected. Their total RNA was extracted by using TRIzol (Invitrogen). Purified RNA with an A260/A280 ratio of 1.8-2.0 was used. Briefly, $0.5 \mu \mathrm{g}$ of total extracted RNA was reversely transcribed (iScript, Bio-Rad). Our data indicated that the amplification was specific. There was only one PCR product amplified for each individual set of primers. Control amplification was done either without RT or without RNA. RT-PCRs were conducted by using the PCR Core Kit (Roche) in a DNA Engine Dyad Peltier Thermal Cycler (Bio-Rad). Primers for $\beta$-actin and cops 6 were

F: 5'-AGATCTGGCATCACACCTTC-3';

R: 5'-TCACCAGAGTCCATCACGAT-3' and

F: 5'-TCTGCATCCGCTGGTGATCC-3';

R: 5'-TCCTGTCCCAGTAGCCGTCA-3', respectively.

\section{Protein alignment}

Alignments were generated by the Megalign program of Lasergene 6 .

\section{Acknowledgements}

We would like to thank our lab members for helpful comments and discussions regarding experiments. We are also grateful to the staff of the Zebrafish Facility in IMCB and NHRI for their excellent maintenance of fish stocks. This work was supported by the Agency of Science, Technology and Research ( $A$ *STAR), Singapore, the National Health Research Institutes, Taiwan (MG-099-PP-13 and MG-099-PP-14) and a grant from the National Science Council, Taiwan (NSC 99-2321-B-400-001).

\section{References}

ALTSCHUL, S.F., MADDEN, T.L., SCHAFFER, A.A., ZHANG, J., ZHANG, Z. MILLER, W. and LIPMAN, D.J. (1997). Gapped BLAST and PSI-BLAST: a new generation of protein database search programs. Nucleic Acids Res. 25: 33893402.

COPE, G.A., SUH, G.S., ARAVIND, L., SCHWARZ, S.E., ZIPURSKY, S.L., KOONIN, E.V. and DESHAIES, R.J. (2002). Role of predicted metalloprotease motif of Jab1/Csn5 in cleavage of Nedd8 from Cul1. Science 298: 608-611.

EISEN, J.S. and SMITH, J.C. (2008). Controlling morpholino experiments: don't stop making antisense. Development 135: 1735-1743.

HANNEMAN, E., TREVARROW, B., METCALFE, W.K., KIMMEL, C.B. and WESTERFIELD, M. (1988). Segmental pattern of development of the hindbrain and spinal cord of the zebrafish embryo. Development 103: 49-58.

HERSHKO, A. and CIECHANOVER, A. (1998). The ubiquitin system. Annu. Rev. Biochem. 67: 425-479.

HUANG, H., LU, F.I., JIA, S., MENG, S., CAO, Y., WANG, Y., MA, W., YIN, K., WEN Z., PENG, J., THISSE, C., THISSE, B. and MENG, A. (2007). Amotl2 is essential for cell movements in zebrafish embryo and regulates c-Src translocation. Development 134: 979-988.

JOYNER, A.L. and GUILLEMOT, F. (1994). Gene targeting and development of the nervous system. Curr Opin Neurobio/4: 37-42.

KEYNES, R. and KRUMLAUF, R. (1994). Hox genes and regionalization of the nervous system. Annu Rev Neurosci 17: 109-132.

KIMMEL, C.B., BALLARD, W.W., KIMMEL, S.R., ULLMANN, B. and SCHILLING, T.F. (1995). Stages of embryonic development of the zebrafish. Dev Dyn203: 253-310

KLEE, E.W., SHIM, K.J., PICKART, M.A., EKKER, S.C. and ELLIS, L.B. (2005) AMOD: a morpholino oligonucleotide selection tool. Nucleic Acids Res 33: W506-511.

KOVALENKO, A., CHABLE-BESSIA, C., CANTARELLA, G., ISRAEL, A., WALLACH, D. and COURTOIS, G. (2003). The tumour suppressor CYLD negatively regulates NF-JB signalling by deubiquitination. Nature 424: 801-805.

KRAUSS, S., JOHANSEN, T., KORZH, V. and FJOSE, A. (1991). Expression of the zebrafish paired box gene pax[zf-b]during early neurogenesis. Development 113: 1193-1206.

MA, M. and JIANG, Y.-J. (2007). Jagged2a-Notch signaling mediates cell fate choice in zebrafish pronephric duct. PLoS Genet. 3: e18.

MARIN, F. and PUELLES, L. (1994). Patterning of the embryonic avian midbrain after experimental inversions: a polarizing activity from the isthmus. Dev Biol 163: 19-37.

MOENS, C.B. and PRINCE, V.E. (2002). Constructing the hindbrain: insights from the zebrafish. Dev Dyn 224: 1-17.

MULLINS, M.C., HAMMERSCHMIDT, M., KANE, D.A., ODENTHAL, J., BRAND, M., VAN EEDEN, F.J., FURUTANI-SEIKI, M., GRANATO, M., HAFFTER, P., HEISENBERG, C.-P., JIANG, Y.-J., KELSH, R.N. and NÜSSLEIN-VOLHARD, C. (1996). Genes establishing dorsoventral pattern formation in the zebrafish embryo: the ventral specifying genes. Development 123: 81-93.

NIJMAN, S.M., LUNA-VARGAS, M.P., VELDS, A., BRUMMELKAMP, T.R., DIRAC A.M., SIXMA, T.K. and BERNARDS, R. (2005). A genomic and functional inventory of deubiquitinating enzymes. Cel/123: 773-786

SCHEEL, H., TOMIUK, S. and HOFMANN, K. (2003). Elucidation of ataxin-3 and ataxin-7 function by integrative bioinformatics. Hum Mol Genet 12: 2845-2852.

TOPCZEWSKI, J., SEPICH, D.S., MYERS, D.C., WALKER, C., AMORES, A., LELE, Z., HAMMERSCHMIDT, M., POSTLETHWAIT, J. and SOLNICA-KREZEL, L. (2001). The zebrafish glypican knypek controls cell polarity during gastrulation movements of convergent extension. Dev Cel/1: 251-264.

TSE, W.K., EISENHABER, B., HO, S.H., NG, Q., EISENHABER, F. and JIANG, Y.J. (2009). Genome-wide loss-of-function analysis of deubiquitylating enzymes for zebrafish development. BMC Genomics 10: 637 


\section{Further Related Reading, published previously in the Int. J. Dev. Biol.}

See our recent Special Issue Placenta edited by Joan S. Hunt and Kent L. Thornburg at: http://www.ijdb.ehu.es/web/contents.php?vol=54\&issue=2-3

A novel role for Glucocorticoid-Induced TNF Receptor Ligand (Gitrl) in early embryonic zebrafish development D. Poulton, Kathleen F. Nolan, Corina Anastasaki, Herman Waldmann and E. Elizabeth Patton Int. J. Dev. Biol. (2010) 54: 815-825

Both jnk and apoptosis pathways regulate growth and terminalia rotation during Drosophila genital disc development Sergio Benitez, Claudia Sosa, Nicolás Tomasini and Ana Macías Int. J. Dev. Biol. (2010) 54: 643-653

Insulin-like growth factor-2 regulates early neural and cardiovascular system development in zebrafish embryos Lori Hartnett, Catherine Glynn, Catherine M. Nolan, Maura Grealy and Lucy Byrnes Int. J. Dev. Biol. (2010) 54: 573-583

Intraovarian transplantation of stage I-II follicles results in viable zebrafish embryos

Zsolt Csenki, Andreas Zaucker, Balázs Kovács, Yavor Hadzhiev, Árpád Hegyi, Katalin-Kinga Lefler, Tamás Muller, Róbert Kovács, Béla Urbányi László Váradi and Ferenc Muller

Int. J. Dev. Biol. (2010) 54: 585-589

The mob as tumor suppressor (mats1) gene is required for growth control in developing zebrafish embryos Yuan Yuan, Shuo Lin, Zuoyan Zhu, Wenxia Zhang and Zhi-Chun Lai Int. J. Dev. Biol. (2009) 53: 525-533

A critical role for myoglobin in zebrafish development Danielle H. Vlecken, Janwillem Testerink, Elisabeth B. Ott, Philippe A. Sakalis, Richard T. Jaspers and Christoph P. Bagowski Int. J. Dev. Biol. (2009) 53: 517-524

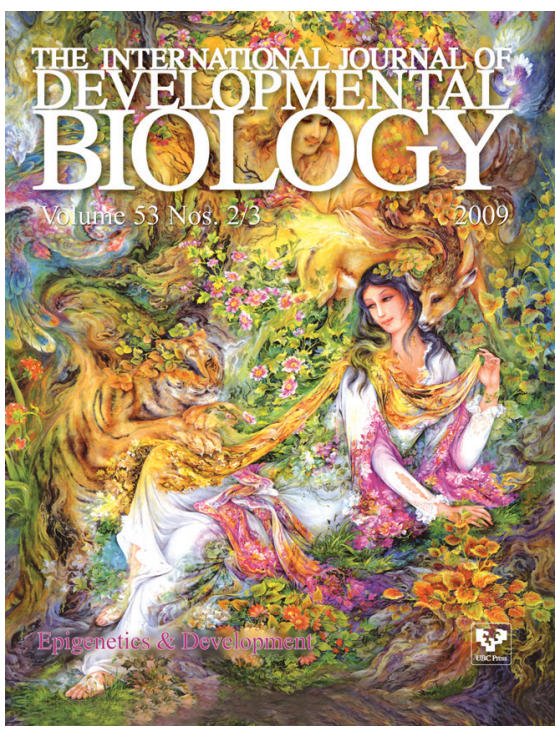

5 yr ISI Impact Factor $(2009)=3.253$

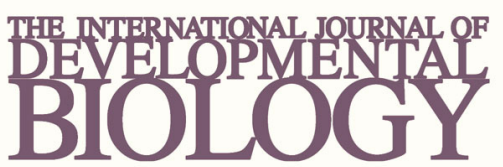

Volume 54 Nos. $6 / 7$
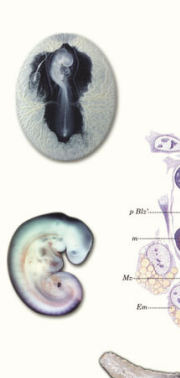

Developmental Hematopoiesis
Special Issue
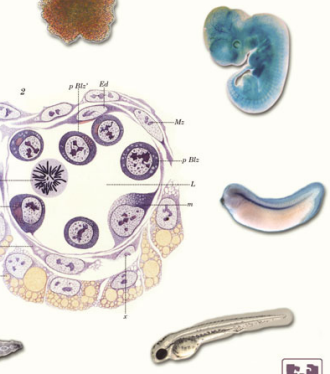

and

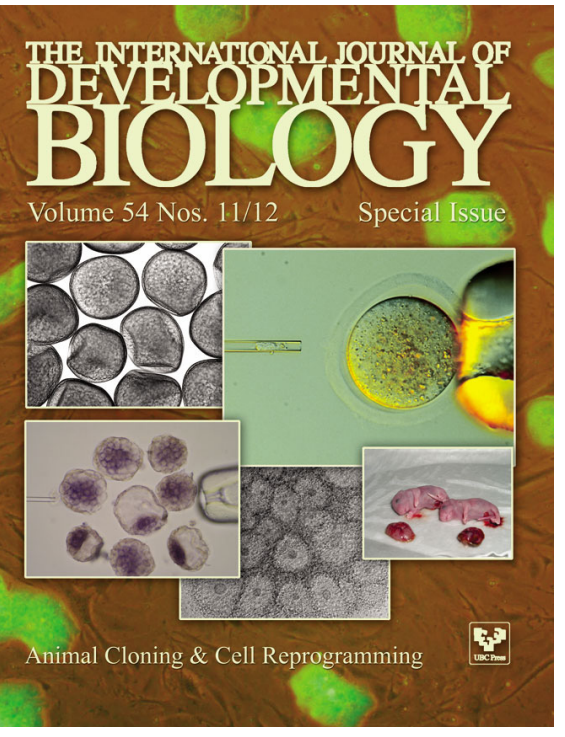

